\title{
Analysis and Improvement on an Authentication Protocol for IoT-Enabled Devices in Distributed Cloud Computing Environment
}

\author{
Baoyuan Kang $(\mathbb{D}$, Yanbao Han, Kun Qian, and Jianqi Du \\ School of Computer Science and Technology, Tiangong University, Tianjin 300387, China \\ Correspondence should be addressed to Baoyuan Kang; baoyuankang@aliyun.com
}

Received 27 November 2019; Accepted 28 May 2020; Published 23 June 2020

Academic Editor: Haipeng Peng

Copyright (c) 2020 Baoyuan Kang et al. This is an open access article distributed under the Creative Commons Attribution License, which permits unrestricted use, distribution, and reproduction in any medium, provided the original work is properly cited.

\begin{abstract}
Recently, a number of authentication protocols integrated with the Internet of Things (IoT) and cloud computing have been proposed for secure access control on large-scale IoT networks. In this paper, we carefully analyze Amin et al.'s authentication protocol for IoT-enabled devices in distributed cloud computing environment and find that Amin et al.'s protocol is vulnerable to several weaknesses. The main shortcoming of Amin et al.'s protocol is in authentication phase; a malicious cloud server can counterfeit the cloud server chosen by a user, and the control server cannot find this counterfeit. To overcome the shortcomings of Amin et al.'s protocol, we propose an improved protocol. In the registration phase of the improved protocol, the pseudoidentity and real identity of a user or a cloud server are bundled up with the control server's secret numbers. This measure can effectively prevent impersonation attack. We also compare the improved protocol with several existing authentication protocols in security and computational efficiency.
\end{abstract}

\section{Introduction}

With the development of the Internet technology, people's life and production have been greatly improved by Internet of Things (IoT) [1]. But, IoT also faces problems of efficiency due to its sensors with low memory and low power. Using powerful cloud services [2] can improve the efficiency of IoT. Now, authentication protocols integrated with the IoT and cloud computing attract people's attention.

The first suggested authentication protocol was proposed by Lamport [3]. Then, many password-based authentication protocols were proposed [4-8]. Recently, people discuss authentication protocols for multiserver in IoT and cloud environment [9-16]. Amin et al. [13] showed security vulnerabilities of two authentication protocols in multiserver cloud environment proposed by Xue et al. [11] and Chuang and Chen [12]. Then, Amin et al. [13] proposed an authentication protocol for IoT-enabled devices in distributed cloud computing environment. They claimed that the proposed protocol is protected against all possible security threats. However, in this paper, we find that Amin et al.'s protocol is vulnerable to several weaknesses. Firstly, during the registration phase of Amin et al.'s protocol, it is unreasonable for a user to register with a pseudoidentity. Secondly, the main shortcoming of Amin et al.'s protocol is that, in its authentication and key agreement phase, although the control server can identify a cloud server is legal, the control server cannot tell if this cloud server is the one chosen by a user. So, in Amin et al.'s protocol, a malicious server can counterfeit the server chosen by a user.

On the basis of analyzing the shortcomings of Amin et al.'s protocol, we propose an improvement on Amin et al.'s protocol. In the registration phase of the improved protocol, the pseudoidentity and real identity of a user or a cloud server are bundled up with the control server's secret numbers. This measure can effectively prevent impersonation attacks. We also compare the improved protocol with two existing protocols $[11,12]$ in security and computational efficiency. 
The rest of the paper is organized as follows. In Section 2, we briefly review Amin et al.'s protocol and analyze its weaknesses. The improved protocol is proposed in Section 3. Security cryptanalysis and comparisons are given in Section 4. Finally, the article is concluded in Section 5.

\section{Amin et al.'s Protocol and Its Weaknesses}

This section briefly reviews the Amin et al.'s protocol [13] and shows its weaknesses. In Amin et al.'s protocol, there are three types of entity such as user $U_{i}$, service provider server $S_{m}$, and control server (CS). The CS is a trusted third party responsible for registration and authentication of users and service providing servers. The $S_{m}$ provides set of services to $U_{i}$. The notations used in this article are recorded in Table 1.

2.1. Amin et al.'s Protocol. Amin et al.'s protocol [13] contains five phases: registration, login, authentication and key agreement, password change, and identity update. For the sake of brevity, password change and identity update phases are not revised.

2.1.1. Registration Phase. During cloud server registration, the cloud server $S_{m}$ sends $\left(\operatorname{SID}_{m}, d\right)$ to CS. After receiving it, the CS computes $\operatorname{PSID}_{m}=h\left(\operatorname{SID}_{m} \| d\right)$ and $\mathrm{BS}_{m}=$ $h\left(\mathrm{PSID}_{m} \| y\right)$ and sends $\mathrm{BS}_{m}$ to $S_{m}$ securely. Finally, $S_{m}$ stores secret parameter $\left(\mathrm{BS}_{m}, d\right)$ into his memory.

During user registration, the user $U_{i}$ computes $A_{i}=h\left(P_{i} \| b_{1}\right)$ and $\operatorname{PID}_{i}=h\left(\operatorname{ID}_{i} \| b_{2}\right) b b_{i}=b_{2} \oplus A_{i}$ and sends $\left(A_{i}, \mathrm{PID}_{i}\right)$ to the CS securely. On getting $\left(A_{i}, \mathrm{PID}_{i}\right)$, the CS calculates $C_{i}=h\left(A_{i} \| \mathrm{PID}_{i}\right), D_{i}=h\left(\mathrm{PID}_{i} \| x\right)$, and $E_{i}=D_{i} \oplus A_{i}$. Finally, the CS prepares and delivers a smartcard for each $U_{i}$ after recording $\left(C_{i}, E_{i}, h(\cdot)\right)$ in the smartcard and transports it to $U_{i}$ through private communication. After getting it, $U_{i}$ records $\left(\mathrm{DP}, b b_{i}\right)$ in the smartcard, where $\mathrm{DP}=h\left(\mathrm{ID}_{i} \| P_{i}\right) \oplus b_{1}$. Finally, the smartcard holds $\left(C_{i}, E_{i}\right.$, DP $\left., b b_{i}, h(\cdot)\right)$.

2.1.2. Login Phase. For accessing server resources, a legal user $U_{i}$ first punches the smartcard into card reader and inputs $\mathrm{ID}_{i}^{*}$ and $P_{i}^{*}$ to the terminal. Then, the card reader calculates $b_{1}^{*}=\mathrm{DP} \oplus h\left(\mathrm{ID}_{i}^{*} \| P_{i}^{*}\right), A_{i}^{*}=h\left(P_{i}^{*} \| b_{i}^{*}\right), \quad b_{2}^{*}=$ $b b_{i}^{*} \oplus A_{i}^{*}, \quad \mathrm{PID}_{i}^{*}=h\left(\mathrm{ID}_{i}^{*} \| b_{2}^{*}\right)$, and $C_{i}^{*}=h\left(A_{i}^{*} \| \mathrm{PID}_{i}^{*}\right)$. Then, the card reader checks the condition $C_{i}^{*}=C_{i}$. If $C_{i}^{*}=C_{i}$, it means that $\mathrm{ID}_{i}^{*}=\mathrm{ID}_{i}$ and $P_{i}^{*}=P_{i}$. The card reader produces a random number $N_{i}$ and computes $D_{i}=E_{i} \oplus A_{i}, \quad G_{i}=h\left(\operatorname{PID}_{i}\left\|\operatorname{SID}_{m}\right\| N_{i}\left\|\mathrm{TS}_{i}\right\| D_{i}\right), \quad F_{i}=D_{i} \oplus$ $N_{i}$, and $Z_{i}=\operatorname{SID}_{m} \oplus h\left(D_{i} \| N_{i}\right)$, where $\operatorname{SID}_{m}$ is the cloud server's identity chosen by the user $U_{i}$. Then, the CR transmits the login messages $\left(G_{i}, F_{i}, Z_{i}, \mathrm{PID}_{i}, \mathrm{TS}_{i}\right)$ to $S_{m}$ publicly.

2.1.3. Authentication and Key Agreement Phase. This phase is necessary for performing mutual authentication as well as key agreement among $U_{i}, S_{m}$, and CS. The detail explanation of this phase is as follows:
TABle 1: Notations table.

\begin{tabular}{lc}
\hline Symbol & Description \\
\hline $\mathrm{CS}$ & The control server \\
$S_{m}$ & $m$ th cloud server \\
$\mathrm{SID}_{m}$ & Identity of the $m$ th cloud server \\
$d$ & Random number of $S_{m}$ \\
$U_{i}$ & ith user \\
$\mathrm{ID}_{i}$ & Identity of the user $U_{i}$ \\
$P_{i}$ & Password of the user $U_{i}$ \\
$b_{1}, b_{2}$ & Two random numbers of $U_{i}$ \\
$x, y$ & Secret numbers of CS \\
$h(\cdot)$ & Hash function \\
$T$ & Timestamp \\
$\oplus$ & Bit-wise xor operation \\
$\|$ & Concatenate operation \\
\hline
\end{tabular}

Step 1: the $S_{m}$ first checks the condition whether $\mathrm{TS}_{m}-$ $\mathrm{TS}_{i}<\Delta T$ holds or not on receiving the login message, where $\mathrm{TS}_{m}$ and $\Delta T$ are the cloud server's current timestamp and expected valid time interval for transmission delay, respectively. If the condition is not true, the $S_{m}$ terminates the connection; otherwise, the $S_{m}$ produces a random number $N_{m}$ and computes $J_{i}=$ $\mathrm{BS}_{m} \oplus N_{m}$ and $K_{i}=h\left(N_{m}\left\|\mathrm{BS}_{m}\right\| G_{i} \| \mathrm{TS}_{m}\right)$. Finally, $S_{m}$ sends $\left(J_{i}, K_{i}, \mathrm{PSID}_{m}, G_{i}, F_{i}, Z_{i}, \mathrm{PID}_{i}, \mathrm{TS}_{i}, \mathrm{TS}_{m}\right)$ to the CS publicly.

Step 2: on getting messages from $S_{m}$, CS first checks the time interval, i.e., $\mathrm{TS}_{\mathrm{CS}}-\mathrm{TS}_{m}<\Delta T$, where $\mathrm{TS}_{\mathrm{CS}}$ and $\Delta T$ are the CS's current timestamp and expected valid time interval for transmission delay, respectively. If the verification holds, CS computes $D_{i}=h\left(\mathrm{PID}_{i} \| x\right)$, $N_{i}^{*}=F_{i} \oplus D_{i}, \quad \operatorname{SID}_{m}^{*}=Z_{i} \oplus h\left(D_{i} \| N_{i}^{*}\right), \quad$ and $G_{i}^{*}=$ $h\left(\mathrm{PID}_{i}\left\|\mathrm{SID}_{m}^{*}\right\| N_{i}^{*}\left\|\mathrm{TS}_{i}\right\| D_{i}\right)$. After that, the CS checks the condition $G_{i}^{*}=G_{i}$. If $G_{i}^{*}=G_{i}$, the CS thinks that the $U_{i}$ is legal; otherwise, the procedures are terminated. After that, the $\mathrm{CS}$ computes $\mathrm{BS}_{m}^{*}=$ $h\left(\mathrm{PSID}_{m} \| y\right), N_{m}^{*}=\mathrm{BS}_{m}^{*} \oplus J_{i}$, and $K_{i}^{*}=h\left(\mathrm{BS}_{m}^{*} \| N_{m}^{*}\right.$ $\left.\left\|G_{i}\right\| \mathrm{TS}_{m}\right)$. Again, the CS checks the condition $K_{i}^{*}=$ $K_{i}$. If $K_{i}^{*}=K_{i}$, the CS thinks that $S_{m}$ is legal; otherwise, the procedure is terminated. After that, the CS chooses a random number $N_{\mathrm{CS}}$ and computes $P_{C S}=$ $N_{m} \oplus N_{C S} \oplus h\left(N_{i} \| D_{i}\right), R_{C S}=N_{i} \oplus N_{C S} \oplus h\left(B S_{m}^{*} \| N_{m}^{*}\right)$, $\mathrm{SK}_{\mathrm{CS}}=h\left(N_{i} \oplus N_{m} \oplus N_{\mathrm{CS}}\right), Q_{\mathrm{CS}}=h\left(\left(N_{m} \oplus N_{\mathrm{CS}}\right) \| \mathrm{SK}_{\mathrm{CS}}\right)$, and $V_{\mathrm{CS}}=h\left(\left(N_{i} \oplus N_{\mathrm{CS}}\right) \| \mathrm{SK}_{\mathrm{CS}}\right)$, where $\mathrm{SK}_{\mathrm{CS}}$ is the secret session key. Finally, the CS sends $\left(P_{\mathrm{CS}}, R_{\mathrm{CS}}, Q_{\mathrm{CS}}\right.$, $V_{\mathrm{CS}}$ ) to $S_{m}$ for achieving mutual authentication of the protocol through public communication.

Step 3: on getting reply messages from CS, $S_{m}$ computes $W_{m}=h\left(B S_{m} \| N_{m}\right), \quad N_{i} \oplus N_{C S}=R_{C S} \oplus W_{m}, \quad S K_{m}=$ $h\left(N_{i} \oplus N_{C S} \oplus N_{m}\right)$, and $V_{\mathrm{CS}}^{*}=h\left(\left(N_{i} \oplus N_{\mathrm{CS}}\right) \| \mathrm{SK}_{m}\right)$. Then, the $S_{m}$ checks the condition $V_{\mathrm{CS}}^{*}=V_{\mathrm{CS}}$ or not. If $V_{\mathrm{CS}}^{*}=V_{\mathrm{CS}}$, the session is terminated; otherwise, messages $\left(P_{\mathrm{CS}}, Q_{\mathrm{CS}}\right)$ are sent to the $U_{i}$ publicly.

Step 4: on obtaining messages from $S_{m}$, the $U_{i}$ calculates $L_{i}=h\left(N_{i} \| D_{i}\right), \quad N_{m} \oplus N_{\mathrm{CS}}=P_{\mathrm{CS}} \oplus L_{i}, \quad \mathrm{SK}_{i}=h\left(N_{m} \oplus\right.$ $\left.N_{\mathrm{CS}} \oplus N_{i}\right)$, and $\mathrm{Q}_{\mathrm{CS}}^{*}=h\left(\left(N_{m} \oplus N_{\mathrm{CS}}\right) \| \mathrm{SK}_{i}\right)$. Then, the $U_{i}$ checks the condition $Q_{\mathrm{CS}}^{*}=Q_{\mathrm{CS}}$, and if $Q_{\mathrm{CS}}^{*}=Q_{\mathrm{CS}}$, it 
proves the authenticity of $S_{m}$ and CS. Finally, the proposed protocol achieves mutual authentication among $U_{i}, S_{m}$, and CS. Now, the $U_{i}$ and the $S_{m}$ can exchange their secret information securely using $\mathrm{SK}_{m}=\mathrm{SK}_{i}$.

2.2. The Weaknesses of Amin et al.'s Protocol. This section shows that Amin et al.'s protocol [13] has some security drawbacks.

2.2.1. Weaknesses in User Registration Phase. During registration in CS, the user $U_{i}$ sends $\left(A_{i}, \mathrm{PID}_{i}\right)$ to the CS. But, $A_{i}=h\left(P_{i} \| b_{i}\right)$ and $\mathrm{PID}_{i}=h\left(\operatorname{ID}_{i} \| b_{2}\right)$.

$\mathrm{PID}_{i}$ is just a pseudoidentity. It is unreasonable for a user to register with a pseudoidentity.

2.2.2. Weaknesses in Authentication and Key Agreement Phase. In authentication and key agreement phase, when the CS receives messages $\left(J_{i}, K_{i}, \operatorname{PSID}_{m}, G_{i}, F_{i}, Z_{i}\right.$, $\mathrm{PID}_{i}, \mathrm{TS}_{i}, \mathrm{TS}_{m}$ ) from the cloud server $S_{m}$, although CS can know the identity $\operatorname{SID}_{m}$ of the server chosen by the user from following calculation,

$$
\begin{aligned}
D_{i} & =h\left(\mathrm{PID}_{i} \| x\right), \\
N_{i} & =F_{i} \oplus D_{i}, \\
\mathrm{SID}_{m} & =Z_{i} \oplus h\left(D_{i} \| N_{i}\right), \\
G_{i}^{*} & =h\left(\mathrm{PID}_{i}\left\|\operatorname{SID}_{m}\right\| N_{i}\left\|\mathrm{TS}_{i}\right\| D_{i}\right),
\end{aligned}
$$

and verifying $G_{i}^{*}=G_{i}$.

CS also can know the server with pseudoidentity $\operatorname{PSID}_{m}$, and the secret value $\mathrm{BS}_{m}$ is a legal server by following calculation:

$$
\begin{aligned}
\mathrm{BS}_{m} & =h\left(\operatorname{PSID}_{m} \| y\right), \\
N_{m} & =\mathrm{BS}_{m} \oplus J_{i}, \\
K_{i}^{*} & =h\left(\mathrm{BS}_{m}\left\|N_{m}\right\| G_{i} \| \mathrm{TS}_{m}\right),
\end{aligned}
$$

and verifying $K_{i}^{*}=K_{i}$.

But, the CS cannot tell if the server with pseudoidentity $\operatorname{PSID}_{m}$ and the secret value $\mathrm{BS}_{m}$ is the one the user chose with real identity SID $_{m}$.

Due to the above weaknesses, a malicious server can counterfeit the server chosen by the user, and the CS cannot see through him.

2.2.3. Puzzling Question of the User. Due to the weaknesses in Section 2.2.2, the user cannot be convinced that the session key $\mathrm{SK}_{i}$ is shared with his chosen server.

\section{The Improved Protocol}

To overcome the shortcomings of Amin et al.'s protocol, in this section, an improved protocol is proposed. Also, for the sake of brevity, only the registration, login, and authentication key agreement phases are described.
3.1. Registration Phase. Suppose the control server CS is a trusted third party responsible for registration and authentication of users and cloud servers. CS chooses two random secret numbers $x$ and $y$.

In registration phase, any cloud server and user can register with CS. When one cloud server $S_{m}$ wants register with $\mathrm{CS}$, it chooses its identity $\mathrm{SID}_{m}$ and a random number $d$. Then, it sends $\left(\operatorname{SID}_{m}, d\right)$ to the control server CS. After CS receives $\left(\operatorname{SID}_{m}, d\right)$, CS computes

$$
\begin{aligned}
\operatorname{PSID}_{m} & =h\left(\operatorname{SID}_{m} \| d\right), \\
\mathrm{BS}_{m} & =h\left(\operatorname{PSID}_{m}\left\|\operatorname{SID}_{m}\right\| y\right),
\end{aligned}
$$

and sends $\mathrm{BS}_{m}$ to the cloud server $S_{m}$ through the secure channel. Once $S_{m}$ receives $\mathrm{BS}_{m}, S_{m}$ stores secret parameters $\left(\mathrm{BS}_{m}, d\right)$.

When one user $U_{i}$ registers with CS, $U_{i}$ chooses his identity $\mathrm{ID}_{i}$ and password $P_{i}$. Then, $U_{i}$ calculates $A_{i}=P_{i} \oplus h\left(B_{i}\right)$. Here, $B_{i}$ is his biometric. Finally, $U_{i}$ submits $\left(\mathrm{ID}_{i}, A_{i}\right)$ to the CS through the secure channel. On receiving the message, CS chooses a random number $b_{i}$ and computes

$$
\begin{aligned}
\mathrm{PID}_{i} & =h\left(\operatorname{ID}_{i} \| b_{i}\right), \\
C_{i} & =h\left(\operatorname{ID}_{i} \| A_{i}\right), \\
D_{i} & =h\left(\operatorname{PID}_{i} \| x\right), \\
E_{i} & =D_{i} \oplus A_{i}, \\
\Delta_{i} & =h\left(\mathrm{PID}_{i}\left\|\operatorname{ID}_{i}\right\| x\right), \\
\Omega_{i} & =b_{i} \oplus A_{i},
\end{aligned}
$$

and issues a smart card containing the information $\left(C_{i}, \Omega_{i}, \Delta_{i}, E_{i}, h(\cdot)\right)$ to the user $U_{i}$.

3.2. Login Phase. After punching his smart card, a user $U_{i}$ provides $\mathrm{ID}_{i}^{*}, P_{i}^{*}$, and $B_{i}^{*}$ to the card reader. The card reader computes

$$
\begin{aligned}
& A_{i}^{*}=P_{i}^{*} \oplus h\left(B_{i}^{*}\right), \\
& C_{i}^{*}=h\left(\mathrm{ID}_{i}^{*} \| A_{i}^{*}\right) .
\end{aligned}
$$

Then, the card reader checks whether $C_{i}^{*}=C_{i}$ or not. When $C_{i}^{*}=C_{i}, \mathrm{ID}_{i}^{*}=\mathrm{ID}_{i}, P_{i}^{*}=P_{i}$, and $B_{i}^{*}=B_{i}, A_{i}^{*}=A_{i}$. Then, the card reader chooses a random number $N_{i}$ and computes

$$
\begin{aligned}
b_{i} & =\Omega_{i} \oplus A_{i}, \\
\mathrm{PID}_{i} & =h\left(\operatorname{ID}_{i} \| b_{i}\right), \\
D_{i} & =E_{i} \oplus A_{i}, \\
O_{i} & =\mathrm{ID}_{i} \oplus D_{i}, \\
G_{i} & =h\left(\operatorname{ID}_{i}\left\|\operatorname{SID}_{m}\right\| N_{i}\left\|\mathrm{TS}_{i}\right\| D_{i}\right), \\
F_{i} & =\Delta_{i} \oplus N_{i}, \\
Z_{i} & =\operatorname{SID}_{m} \oplus h\left(D_{i} \| N_{i}\right),
\end{aligned}
$$


where $\operatorname{SID}_{m}$ is the identity of the cloud server $S_{m}$ chosen by the user $U_{i}$. Then, the card reader sends the login messages $\left(G_{i}, F_{i}, Z_{i}, O_{i}, \mathrm{PID}_{\mathrm{i}}, \mathrm{TS}_{i}\right)$ to the cloud server $S_{m}$ publicly. $\mathrm{TS}_{i}$ is the $U_{i}$ 's current timestamp.

3.3. Authentication Key Agreement Phase. This phase includes four steps. It is also illustrated in Figure 1.

Step 1: once $S_{m}$ receives the login message, $S_{m}$ checks the condition whether $\mathrm{TS}_{m}-\mathrm{TS}_{i}<\Delta T$ holds or not. If the condition is true, $S_{m}$ chooses a random number $N_{m}$ and computes

$$
\begin{aligned}
J_{i} & =\mathrm{BS}_{m} \oplus N_{m}, \\
K_{i} & =h\left(N_{m}\left\|\mathrm{BS}_{m}\right\| \mathrm{PID}_{i}\left\|G_{i}\right\| \mathrm{TS}_{m}\right) .
\end{aligned}
$$

Then, the $S_{m}$ submits $\left(J_{i}, K_{i}, \operatorname{PSID}_{m}, G_{i}, F_{i}, Z_{i}, O_{i}\right.$, $\mathrm{PID}_{i}, \mathrm{TS}_{i}, \mathrm{TS}_{m}$ ) to the CS. Here, $\mathrm{TS}_{m}$ and $\Delta T$ are the cloud server's current timestamp and expected time interval for transmission delay, respectively.

Step 2: on receiving the messages from $S_{m}$, CS first checks whether $\mathrm{TS}_{\mathrm{CS}}-\mathrm{TS}_{m}<\Delta \mathrm{T}$ holds or not, where $\mathrm{TS}_{\mathrm{CS}}$ and $\Delta T$ are the similar meanings mentioned before. If the verification holds, CS calculates

$$
\begin{aligned}
D_{i} & =h\left(\mathrm{PID}_{i} \| x\right), \\
\mathrm{ID}_{i} & =O_{i} \oplus D_{i}, \\
N_{i} & =F_{i} \oplus h\left(\mathrm{PID}_{i}\left\|\mathrm{ID}_{i}\right\| x\right), \\
\operatorname{SID}_{m} & =Z_{i} \oplus h\left(D_{i} \| N_{i}\right), \\
G_{i}^{*} & =h\left(\operatorname{ID}_{i}\left\|\operatorname{SID}_{m}\right\| N_{i}\left\|\mathrm{TS}_{i}\right\| D_{i}\right) .
\end{aligned}
$$

Then, the CS checks whether $G_{i}^{*}=G_{i}$ holds or not. If $G_{i}^{*}=G_{i}$, the CS believes the $U_{i}$ with real identity $\operatorname{ID}_{i}$ is legal. Then, the CS computes

$$
\begin{aligned}
\mathrm{BS}_{m} & =h\left(\operatorname{PSID}_{m}\left\|\operatorname{SID}_{m}\right\| y\right), \\
N_{m} & =\mathrm{BS}_{m} \oplus J_{i}, \\
K_{i}^{*} & =h\left(N_{m}\left\|\mathrm{BS}_{m}\right\| \mathrm{PID}_{i}\left\|G_{i}\right\| \mathrm{TS}_{m}\right) .
\end{aligned}
$$

Then, the CS checks the condition $K_{i}^{*}=K_{i}$. If $K_{i}^{*}=K_{i}$, the CS believes $S_{m}$ with real $\operatorname{SID}_{m}$ is legal and chosen by the user $U_{i}$. Then, the CS produces a random number $N_{\text {CS }}$ and computes

$$
\begin{aligned}
P_{\mathrm{CS}} & =N_{m} \oplus N_{\mathrm{CS}} \oplus h\left(N_{i}\left\|D_{i}\right\| F_{i}\right), \\
R_{\mathrm{CS}} & =N_{i} \oplus N_{\mathrm{CS}} \oplus h\left(\mathrm{BS}_{m} \| N_{m}\right), \\
\mathrm{SK}_{\mathrm{CS}} & =h\left(N_{i} \oplus N_{m} \oplus N_{\mathrm{CS}}\right), \\
Q_{\mathrm{CS}} & =h\left(\left(N_{m} \oplus N_{\mathrm{CS}}\right) \| \mathrm{SK}_{\mathrm{CS}}\right), \\
V_{\mathrm{CS}} & =h\left(\left(N_{i} \oplus N_{\mathrm{CS}}\right) \| \mathrm{SK}_{\mathrm{CS}}\right) .
\end{aligned}
$$

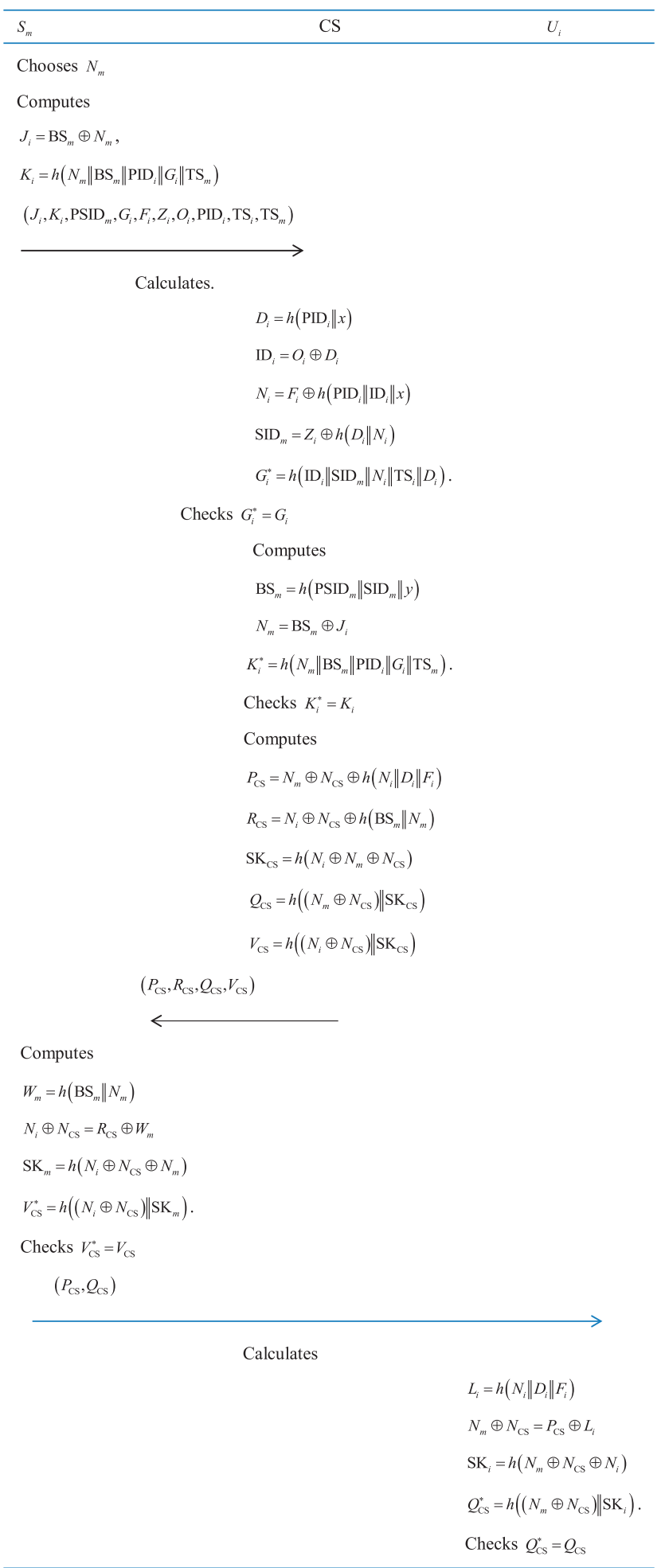

FIgURE 1: Authentication key agreement phase.

Then, the CS sends $\left(P_{\mathrm{CS}}, R_{\mathrm{CS}}, Q_{\mathrm{CS}}, V_{\mathrm{CS}}\right)$ to the $S_{m}$ publicly.

Step 3: on receiving the reply messages from CS, $S_{m}$ computes 


$$
\begin{aligned}
W_{m} & =h\left(\mathrm{BS}_{m} \| N_{m}\right), \\
N_{i} \oplus N_{\mathrm{CS}} & =R_{\mathrm{CS}} \oplus W_{m}, \\
\mathrm{SK}_{m} & =h\left(N_{i} \oplus N_{\mathrm{CS}} \oplus N_{m}\right), \\
V_{\mathrm{CS}}^{*} & =h\left(\left(N_{i} \oplus N_{\mathrm{CS}}\right) \| \mathrm{SK}_{m}\right) .
\end{aligned}
$$

Then, the $S_{m}$ checks the condition $V_{\mathrm{CS}}^{*}=V_{\mathrm{CS}}$. If $V_{\mathrm{CS}}^{*}=V_{\mathrm{CS}}, S_{m}$ sends messages $\left(P_{\mathrm{CS}}, Q_{\mathrm{CS}}\right)$ to the $U_{i}$ publicly.

Step 4: on receiving messages from $S_{m}$, the $U_{i}$ calculates

$$
\begin{aligned}
L_{i} & =h\left(N_{i}\left\|D_{i}\right\| F_{i}\right), \\
N_{m} \oplus N_{\mathrm{CS}} & =P_{\mathrm{CS}} \oplus L_{i}, \\
\mathrm{SK}_{i} & =h\left(N_{m} \oplus N_{\mathrm{CS}} \oplus N_{i}\right), \\
\mathrm{Q}_{\mathrm{CS}}^{*} & =h\left(\left(N_{m} \oplus N_{\mathrm{CS}}\right) \| \mathrm{SK}_{i}\right) .
\end{aligned}
$$

Next, the $U_{i}$ checks whether $Q_{C S}^{*}=Q_{C S}$. If $Q_{C S}^{*}=Q_{C S}$, $U_{i}$ believes the authenticity of $S_{m}$ and CS and shares a session key $\mathrm{SK}_{i}\left(=\mathrm{SK}_{m}\right)$ with the cloud server $S_{m}$.

\section{Security Analysis and Comparisons}

4.1. Security Analysis. This section shows that the improved protocol is well protected against relevant security threats. Firstly, like Amin et al.'s protocol [13], the improved protocol is user anonymous and protected against password guessing attack, replay attack, insider attack, and session key discloser attack. For the shortcomings of Amin et al.'s protocol, the following analysis is focused on the improved protocol against impersonation attack.

In cloud server registration phase of the improved protocol, the cloud server $S_{m}$ with identity $\operatorname{SID}_{m}$ and pseudoidentity $\operatorname{PSID}_{m}$ has secret value

$$
\mathrm{BS}_{m}=h\left(\operatorname{PSID}_{m}\left\|\operatorname{SID}_{m}\right\| y\right),
$$

computed by the control server CS. So, in the authentication phase, if one cloud server $S_{m}^{*}$ not chosen by the user $U_{i}$ counterfeits $S_{m}, S_{m}^{*}$ intercepts the login messages $\left(G_{i}, F_{i}, Z_{i}, O_{i}, \mathrm{PID}_{i}, \mathrm{TS}_{i}\right)$ from $U_{i}$ and computes

$$
\begin{aligned}
J_{i} & =\mathrm{BS}_{m}^{*} \oplus N_{m}^{*}, \\
K_{i} & =h\left(N_{m}\left\|\mathrm{BS}_{m}^{*}\right\| \mathrm{PID}_{i}\left\|G_{i}\right\| \mathrm{TS}_{m}^{*}\right),
\end{aligned}
$$

where $\mathrm{BS}_{m}^{*}=h\left(\operatorname{PSID}_{m}^{*}\left\|\operatorname{SID}_{m}^{*}\right\| y\right)$. Then, $S_{m}^{*}$ sends

$$
\left(J_{i}, K_{i}, \operatorname{PSID}_{m}^{*}, G_{i}, F_{i}, Z_{i}, O_{i}, \mathrm{PID}_{i}, \mathrm{TS}_{i}, \mathrm{TS}_{m}^{*}\right),
$$

to the CS publicly. But, CS obtains the identity $\operatorname{SID}_{m}$ of the cloud server $S_{m}$ chosen by the user $U_{i}$ from $Z_{i}$ and computes

$$
\begin{aligned}
\overline{\mathrm{BS}}_{m}^{*} & =h\left(\operatorname{PSID}_{m}^{*}\left\|\operatorname{SID}_{m}\right\| y\right), \\
N_{m}^{*} & =\overline{\mathrm{BS}}_{m}^{*} \oplus J_{i}, \\
K_{i}^{*} & =h\left(N_{m}^{*}\left\|\overline{\mathrm{BS}}_{m}^{*}\right\| \mathrm{PID}_{i}\left\|G_{i}\right\| \mathrm{TS}_{m}^{*}\right) .
\end{aligned}
$$

Obviously, due to $\mathrm{BS}_{m}^{*} \neq \overline{\mathrm{BS}}_{m}^{*}$, then $K_{i}^{*} \neq K_{i}$. So, $S_{m}^{*}$ cannot pass the CS's verification.
TABle 2: Comparison of security.

\begin{tabular}{lccccccc}
\hline & F1 & F2 & F3 & F4 & F5 & F6 & F7 \\
\hline Xue et al. [11] & Yes & No & Yes & Yes & Yes & Yes & Yes \\
Amin et al. [13] & Yes & Yes & Yes & Yes & Yes & No & No \\
Improved protocol & Yes & Yes & Yes & Yes & Yes & Yes & Yes \\
\hline
\end{tabular}

F1: user anonymity; F2: resist password guessing attack; F3: resist replay attack; F4: resist insider attack; F5: resist session key discloser attack; F6: resist impersonation attack; F7: CS knows the real identities of users and cloud servers.

Table 3: Comparison of computation costs.

\begin{tabular}{lcccc}
\hline & $\mathrm{P} 1$ & $\mathrm{P} 2$ & $\mathrm{P} 3$ & $\mathrm{P} 4$ \\
\hline Xue et al. [11] & $7 \mathrm{H}+2 \mathrm{X}$ & $5 \mathrm{H}+5 \mathrm{X}$ & $25 \mathrm{H}+25 \mathrm{X}$ & $37 \mathrm{H}+32 \mathrm{X}$ \\
Improved protocol & $7 \mathrm{H}+3 \mathrm{X}$ & $5 \mathrm{H}+6 \mathrm{X}$ & $18 \mathrm{H}+21 \mathrm{X}$ & $30 \mathrm{H}+30 \mathrm{X}$ \\
\hline P1: registration phase; $\mathrm{P} 2$ : login phase; P3: authentication phase; P4: total \\
computation cost; H: hash computation and its time cost; X: xor operation \\
and its time cost.
\end{tabular}

If $S_{m}^{*}$ wants to tamper $Z_{i}$ by computing $Z_{i}^{*}=$ $\operatorname{SID}_{m}^{*} \oplus h\left(D_{i}^{*} \| N_{i}^{*}\right)$, since $D_{i}^{*} \neq D_{i}$ and $N_{i}^{*} \neq N_{i}, S_{m}^{*}$ also cannot pass the CS's verification.

Therefore, the improved protocol is protected against cloud server impersonation attack.

In Amin et al.'s protocol, CS does not know the real identities of the user. But, in improved protocol, we use $O_{i}$ to show the real identities of the user. Also, $\Delta_{i}=h\left(\mathrm{PID}_{i}\left\|\mathrm{ID}_{i}\right\| x\right)$ is used in the improved protocol, and the user cannot pass CS's verification if he uses false identity.

In summary, the improved protocol completely overcomes the shortcomings of Amin et al.'s protocol. In the improved protocol, neither the user nor the cloud server can launch impersonation attacks. In the improved protocol, the user and the cloud server can use the shared session key between them with trust.

4.2. Comparisons. In this section, the comparison of the improved protocol with other protocols $[11,13]$ is shown. The comparison results of the security features and computation costs are shown, respectively, in Tables 2 and 3.

From Table 2, the improved protocol is superior to the protocols $[11,13]$ in terms of security. Furthermore, in Table 3, the comparison of computation costs is shown between the improved protocol and the relatively good protocol [11]. From Table 3, the total computation cost of the protocol [11] is $37 \mathrm{H}+32 \mathrm{X}$, but the total computational cost of the improved scheme is $30 \mathrm{H}+30 \mathrm{X}$. The computation cost of the improved protocol is significantly less than the protocol [11].

\section{Conclusion}

In this paper, we find that Amin et al.'s authentication protocol is vulnerable to several weaknesses. To overcome the shortcomings of Amin et al.'s protocol, we propose an improved protocol. We also compare the improved protocol with several existing authentication protocols in security and computational efficiency. The improved protocol not only 
completely overcomes the shortcomings of Amin et al.'s protocol but also has less computation cost.

\section{Data Availability}

The data used to support the findings of this study are available from the corresponding author upon request.

\section{Conflicts of Interest}

The authors declare that there are no conflicts of interest regarding the publication of this paper.

\section{Acknowledgments}

This work was supported by the Applied Basic and Advanced Technology Research Programs of Tianjin (no. 15JCYBJC15900) and the National Natural Science Foundation of China (no. 61972456).

\section{References}

[1] L. Atzori, A. Iera, and G. Morabito, "The internet of things: a survey," Computer Networks, vol. 54, no. 15, pp. 2787-2805, 2010.

[2] G. Ateniese, R. Burns, R. Curtmola et al., "Provable data possession at untrusted stores," in Proceedings of the 14th ACM Conference on Computer and Communications Security (CCS'07), pp. 598-609, Virginia, VA, USA, November 2007.

[3] L. Lamport, "Password authentication with insecure communication," Communications of the ACM, vol. 24, no. 11, pp. 770-772, 1981.

[4] A. K. Awashti and S. Lal, "An enhanced remote user authentication scheme using smart cards," IEEE Transaction on Consumer Electronics, vol. 50, no. 2, pp. 583-586, 2004.

[5] M. K. Khan, S. Kumari, and M. K. Gupta, "More efficient keyhash based fingerprint remote authentication scheme using mobile device," Computing, vol. 96, no. 9, pp. 793-816, 2013.

[6] M. Kumar, M. K. Gupta, and S. Kumari, "An improved efficient remote password authentication scheme with smartcard over insecure networks," International Journal of Network Security, vol. 13, no. 3, pp. 167-177, 2011.

[7] B. Kang, J. Han, and Q. Wang, "Cryptanalysis and improvement on an IC-card-based remote login mechanism," in Proceedings of the 2010 2nd International Conference on Computer Engineering and Technology, vol. 1, pp. 65-68, Bali Island, Indonesia, April 2010.

[8] B. Kang and J. Han, "Cryptanalysis and improvement on three-party protocols for password authenticated key exchange," in Proceedings of the 2010 2nd International Conference on Education Technology and Computer, vol. 5, pp. 5197-5201, Shanghai, China, June 2010.

[9] S. K. Sood, A. K. Sarje, and K. Singh, "A secure dynamic identity based authentication protocol for multi-server architecture," Journal of Network and Computer Applications, vol. 34, no. 2, pp. 609-618, 2011.

[10] X. Li, Y. Xiong, J. Ma, and W. Wang, "An efficient and security dynamic identity based authentication protocol for multiserver architecture using smart cards," Journal of Network and Computer Applications, vol. 35, no. 2, pp. 763-769, 2012.

[11] K. Xue, P. Hong, and C. Ma, "A lightweight dynamic pseudonym identity based authentication and key agreement protocol without verification tables for multi-server architecture," Journal of Computer and System Sciences, vol. 80, no. 1, pp. 195-206, 2014.

[12] M.-C. Chuang and M. C. Chen, "An anonymous multi-server authenticated key agreement scheme based on trust computing using smart cards and biometrics," Expert Systems with Applications, vol. 41, no. 4, pp. 1411-1418, 2014.

[13] R. Amin, N. Kumar, G. P. Biswas, R. Iqbal, and V. Chang, "A light weight authentication protocol for IoT-enabled devices in distributed Cloud Computing environment," Future Generation Computer Systems, vol. 78, pp. 1005-1019, 2018.

[14] L. Zhou, X. Li, K. Yeh, C. Su, and W. Chiu, "Lightweight IoTbased authentication scheme in cloud computing circumstance," Future Generation Computer Systems, vol. 91, pp. 1244-1251, 2019.

[15] K.-H. Wang, C.-M. Chen, W. Fang, and T.-Y. Wu, "A secure authentication scheme for internet of things," Pervasive and Mobile Computing, vol. 42, pp. 15-26, 2017.

[16] O. Ruan, N. Kumar, D. He, and J.-H. Lee, "Efficient provably secure password-based explicit authenticated key agreement," Pervasive and Mobile Computing, vol. 24, pp. 50-60, 2015. 